\title{
Estimation of $R_{3}=\operatorname{Pr}\left(Y_{3}>\max \left(Y_{1}, Y_{2}\right)\right)$ for Exponential Distribution
}

\author{
Piyali Kundu \\ Department of Statistics, Visva Bharati University, Santiniketan, India \\ Email: vbpiyali@gmail.com
}

\begin{abstract}
This paper deals with the estimation of the reliability function $R_{3}=\operatorname{Pr}\left(Y_{3}>\max \left(Y_{1}, Y_{2}\right)\right)$, when the random variables $Y_{1}, Y_{2}$ and $Y_{3}$ are independent exponential with respective unknown location parameters $\mu_{1}, \mu_{2}$ and $\mu_{3}$. Here we have derived several estimators of $R_{3}$ which includes uniformly minimum variance unbiased estimator (UMVUE), Bayes estimator (BE) and generalized Bayes estimator(GBE). Moreover, we have compared these estimators based on their risks under different loss functions.
\end{abstract}

Keywords: Stress-strength reliability, UMVUE, Bayes estimator, generalized Bayes estimator.

\section{Introduction}

Stress-strength reliability of a component having strength $X$ subject to a stress $Y$ is defined by $R=$ $\operatorname{Pr}(Y>X)$. Literature review reveals the importance of the estimation of Stress-strength reliability under various lifetime distributions. In this regard mention may be made to Kotz et al. (2003), Baklizi (2008a, 2008b), Eryilmaz (2008a, 2008b, 2010, 2011), Krishnamoorthy et al. (2007, 2009, 2010), Krishnamoorthy and Lin (2010), Kundu and Raqab (2009), Rezaei et al. (2010). Recently, Rao (2012, 2013) studied the estimation of reliability in a multicomponent stress-strength model. Rinco(1983) initiated a study on the estimation of $R_{p}=\operatorname{Pr}\left(Y_{p}>\max \left(Y_{1}, Y_{2}, \cdots, Y_{p-1}\right)\right)$ and provided a particular estimator. Gupta and Gupta (1988) considered a similar problem and provided three other estimators. Unfortunately, some estimators are difficult to derive in general and only the special case of $\mathrm{p}=2$ was found to be explicitly solvable. Literature review also reveals that even for the case $p=3$, no work has yet been done for the case of exponential distribution with different location parameters.

The study of the estimation of stress-strength reliability of a component for exponential lifetime distributions with common scale parameters are important from the theoretical point of view which can be evidenced from Ali et al. (2004), Ivshin (1996), Pal et al. (2005) and Sengupta (2008).

The paper is organized as follows. In Section 2, we provide the notations and preliminaries. Section 3 considers the estimation aspect of $R_{3}$ and Section 4 compares the performances of the estimators through a simulation study.

\section{Notation and Preliminaries}

In this section, we provide the following notations and preliminaries which will be helpful in developing the estimation of the reliability function $R_{3}=\operatorname{Pr}\left(Y_{3}>\max \left(Y_{1}, Y_{2}\right)\right)$, where $Y_{3}$ denotes the strength and $Y_{1}, Y_{2}$ are the stresses. It is assumed that $Y_{1}, Y_{2}, Y_{3}$ are independent random variables having the following probability density functions.

$$
f\left(y_{i}\right)=\exp \left\{-\left(y_{i}-\mu_{i}\right)\right\} ; y_{i} \geq \mu_{i},-\infty<\mu_{i}<\infty, i=1,2,3 .
$$

Defining $T=\max \left(Y_{1}, Y_{2}\right)$ and $\mu=\max \left(\mu_{1}, \mu_{2}\right)$, we can write

$$
R_{3}=\operatorname{Pr}\left(Y_{3}>T\right)=1-\int_{\mu}^{\infty} \operatorname{Pr}\left(Y_{3} \leq t \mid T=t\right) f(t) d t=1-\bar{R}_{3}
$$


where $f(t)$ denotes the probability density function of $T$ which is stated below

$$
f(t)=\sum_{i=1}^{2} \exp \left\{-\left(t-\mu_{i}\right)\right\}-2 \exp \left\{-\left(2 t-\mu_{1}-\mu_{2}\right)\right\}, t \geq \mu .
$$

In order to get an expression for $\bar{R}_{3}$, we consider the following cases.

Case-1: $\mu \leq \mu_{3}$

$$
\begin{aligned}
\bar{R}_{3} & =\int_{\mu_{3}}^{\infty} \operatorname{Pr}\left(Y_{3} \leq t \mid T=t\right) f(t) d t \\
& =\frac{1}{2} \sum_{i=1}^{2} \exp \left\{-\left(\mu_{3}-\mu_{i}\right)\right\}-\frac{1}{3} \exp \left\{-\left(2 \mu_{3}-\mu_{2}-\mu_{1}\right)\right\} \\
& =R_{1}(\text { say })
\end{aligned}
$$

Case-2: $\mu>\mu_{3}$

$$
\begin{aligned}
\bar{R}_{3}= & \int_{\mu}^{\infty} \operatorname{Pr}\left(Y_{3} \leq t \mid T=t\right) f(t) d t \\
= & \sum_{i=1}^{2} \exp \left\{-\left(\mu-\mu_{i}\right)\right\}-\exp \left\{-\left(2 \mu-\mu_{1}-\mu_{2}\right)\right\}- \\
& \frac{1}{2} \exp \left\{-\left(2 \mu-\mu_{1}-\mu_{3}\right)\right\}-\frac{1}{2} \exp \left\{-\left(2 \mu-\mu_{1}-\mu_{3}\right)\right\} \\
& +\frac{2}{3} \exp \left\{-\left(3 \mu-\mu_{1}-\mu_{2}-\mu_{3}\right)\right\} \\
= & R_{2}(\text { say })
\end{aligned}
$$

Thus the reliability function which is defined in (2) can be expressed as

$$
R_{3}=\left\{\begin{array}{l}
1-R_{1} \text { if } \mu \leq \mu_{3} \\
1-R_{2} \text { if } \mu>\mu_{3}
\end{array}\right.
$$

or equivalently,

$$
R_{3}=\left(1-R_{1}\right) I\left(\mu \leq \mu_{3}\right)+\left(1-R_{2}\right) I\left(\mu>\mu_{3}\right)
$$

where $I$ denotes the indicator function. In the next section, we will concentrate on the different estimators of the reliability function as defined in (3).

\section{Estimation}

Let $Y_{i 1}, Y_{i 2}, \cdots, Y_{i n_{i}}$ be a random sample of size $n_{i}$ from the population of $Y_{i}$. Define

$$
Y_{i(1)}=\operatorname{Min}\left(Y_{i 1}, Y_{i 2}, \cdots, Y_{i n_{i}}\right) ; i=1,2,3 .
$$

Throughout this section, these random samples will be used to estimate $R_{3}$.

\subsection{Maximum Likelihood Estimator of $R_{3}$}

It is well known that the MLEs of $\mu_{1}, \mu_{2}$ and $\mu_{3}$, are $\widehat{\mu}_{1 M}, \widehat{\mu}_{2 M}$ and $\widehat{\mu}_{3 M}$, respectively, where $\widehat{\mu}_{i M}=$ $Y_{i(1)}, i=1,2,3$. Then from (3), MLE of $R_{3}$ will be given by

$$
\widehat{R}_{3 M}=\left(1-\widehat{R}_{1 M}\right) I\left(\widehat{\mu}_{M} \leq \widehat{\mu}_{3 M}\right)+\left(1-\widehat{R}_{2 M}\right) I\left(\widehat{\mu}_{M}>\widehat{\mu}_{3 M}\right),
$$


where $\widehat{\mu}_{M}=\max \left(\widehat{\mu}_{1 M}, \widehat{\mu}_{2 M}\right)$,

$$
\widehat{R}_{1 M}=\frac{1}{2} \sum_{i=1}^{2} \exp \left\{-\left(\widehat{\mu}_{3 M}-\widehat{\mu}_{i M}\right)\right\}-\frac{1}{3} \exp \left\{-\left(2 \widehat{\mu}_{3 M}-\widehat{\mu}_{2 M}-\widehat{\mu}_{1 M}\right)\right\},
$$

and

$$
\begin{aligned}
\widehat{R}_{2 M}= & \sum_{i=1}^{2} \exp \left\{-\left(\widehat{\mu}_{M}-\widehat{\mu}_{i M}\right)\right\}-\exp \left\{-\left(2 \widehat{\mu}_{M}-\widehat{\mu}_{2 M}-\widehat{\mu}_{1 M}\right)\right\} \\
& -\frac{1}{2} \exp \left\{-\left(2 \widehat{\mu}_{M}-\widehat{\mu}_{1 M}-\widehat{\mu}_{3 M}\right)\right\}-\frac{1}{2} \exp \left\{-\left(2 \widehat{\mu}_{M}-\widehat{\mu}_{2 M}-\widehat{\mu}_{3 M}\right)\right\} \\
& +\frac{2}{3} \exp \left\{-\left(3 \widehat{\mu}_{M}-\widehat{\mu}_{1 M}-\widehat{\mu}_{2 M}-\widehat{\mu}_{3 M}\right)\right\} .
\end{aligned}
$$

\subsection{Uniformly Minimum Variance Unbiased Estimator of $\boldsymbol{R}_{3}$}

Based on the random sample $Y_{i 1}, Y_{i 2}, \cdots, Y_{i n_{i}}$, let us define

$$
U_{i}=Y_{i(1)}, i=1,2,3 .
$$

In addition, we set $U_{1}=u_{1}, U_{2}=u_{2}, U_{3}=u_{3}$. We now state and prove the following theorem which will be helpful to derive the UMVUE of $R_{3}$.

Theorem 1. Let $T_{1}=\max \left(Y_{11}, Y_{21}\right)$. Then

(i) for, $u_{1}<u_{2}$, the distribution function of $T_{1}$ given $\left(u_{1}, u_{2}\right)$ will be

$$
\begin{aligned}
& F\left(t_{1} \mid\left(u_{1}, u_{2}\right)\right) \\
= & \begin{cases}\frac{1}{n_{2}}\left[1-\frac{n_{1}-1}{n_{1}} \exp \left\{-\left(u_{2}-u_{1}\right)\right\}\right] & \text { if } t_{1}=u_{2} \\
\left(1-\frac{\left(n_{1}-1\right)}{n_{1}} \exp \left\{-\left(t_{1}-u_{1}\right)\right\}\right)\left(1-\frac{\left(n_{2}-1\right)}{n_{2}} \exp \left\{-\left(t_{1}-u_{2}\right)\right\}\right) & \text { if } t_{1}>u_{2}\end{cases}
\end{aligned}
$$

(ii) for, $u_{1}>u_{2}$, the distribution function of $T_{1}$ given $\left(u_{1}, u_{2}\right)$ will be

$$
\begin{aligned}
& F\left(t_{1} \mid\left(u_{1}, u_{2}\right)\right) \\
= & \begin{cases}\frac{1}{n_{1}}\left[1-\frac{n_{2}-1}{n_{2}} \exp \left\{-\left(u_{1}-u_{2}\right)\right\}\right] & \text { if } t_{1}=u_{1} \\
\left(1-\frac{\left(n_{1}-1\right)}{n_{1}} \exp \left\{-\left(t_{1}-u_{1}\right)\right\}\right)\left(1-\frac{\left(n_{2}-1\right)}{n_{2}} \exp \left\{-\left(t_{1}-u_{2}\right)\right\}\right) & \text { if } t_{1}>u_{1} .\end{cases}
\end{aligned}
$$

Proof. From definition, the distribution function of $T_{1}$ given $\left(u_{1}, u_{2}\right)$ is given by

$$
\begin{aligned}
F\left(t_{1} \mid\left(u_{1}, u_{2}\right)\right) & =\operatorname{Pr}\left(T_{1} \leq t_{1} \mid\left(u_{1}, u_{2}\right)\right) \\
& =\operatorname{Pr}\left(\max \left(Y_{11}, Y_{21}\right) \leq t_{1} \mid\left(u_{1}, u_{2}\right)\right) \\
& =\operatorname{Pr}\left(Y_{11} \leq t_{1}, Y_{21} \leq t_{1} \mid\left(u_{1}, u_{2}\right)\right) \\
& =\operatorname{Pr}\left(Y_{11} \leq t_{1} \mid u_{1}\right) \operatorname{Pr}\left(Y_{21} \leq t_{1} \mid u_{2}\right) .
\end{aligned}
$$

It is to be noted that, for $i=1,2,3$,

$$
P\left(Y_{i 1} \leq y_{i 1} \mid u_{i}\right)= \begin{cases}0 & \text { if } y_{i 1}<u_{i} \\ \frac{1}{n_{i}} & \text { if } y_{i 1}=u_{i} \\ 1-\frac{n_{i}-1}{n_{i}} \exp \left\{-\left(y_{i 1}-u_{i}\right)\right\} & \text { if } y_{i 1}>u_{i}\end{cases}
$$

Now, for $u_{1}<u_{2}$, we have

$$
\begin{aligned}
F\left(t_{1} \mid\left(u_{1}, u_{2}\right)\right) & =\operatorname{Pr}\left(Y_{11} \leq t_{1} \mid u_{1}\right) \operatorname{Pr}\left(Y_{21} \leq t_{1} \mid u_{2}\right) \\
& =\left\{\begin{array}{l}
\operatorname{Pr}\left(Y_{11} \leq u_{2} \mid u_{1}\right) \operatorname{Pr}\left(Y_{21} \leq u_{2} \mid u_{2}\right) \text { if } t_{1}=u_{2} \\
\operatorname{Pr}\left(Y_{11} \leq t_{1} \mid u_{1}\right) \operatorname{Pr}\left(Y_{21} \leq t_{1} \mid u_{2}\right) \text { if } t_{1}>u_{2}
\end{array},\right.
\end{aligned}
$$


and, for $u_{1}>u_{2}$, we have

$$
\begin{aligned}
F\left(t_{1} \mid\left(u_{1}, u_{2}\right)\right) & =\operatorname{Pr}\left(Y_{11} \leq t_{1} \mid u_{1}\right) \operatorname{Pr}\left(Y_{21} \leq t_{1} \mid u_{2}\right) \\
& =\left\{\begin{array}{l}
\operatorname{Pr}\left(Y_{11} \leq u_{1} \mid u_{1}\right) \operatorname{Pr}\left(Y_{21} \leq u_{1} \mid u_{2}\right) \text { if } t_{1}=u_{1} \\
\operatorname{Pr}\left(Y_{11} \leq t_{1} \mid u_{1}\right) \operatorname{Pr}\left(Y_{21} \leq t_{1} \mid u_{2}\right) \text { if } t_{1}>u_{1}
\end{array}\right.
\end{aligned}
$$

From (4), the proof of Theorem 1 follows immediately.

Let us now define the following:

$$
\begin{aligned}
I_{1}= & \frac{\left(n_{1}-1\right)\left(n_{3}+1\right)}{2 n_{1} n_{3}} \exp \left\{-\left(u_{3}-u_{1}\right)\right\}+\frac{\left(n_{2}-1\right)\left(n_{3}+1\right)}{2 n_{2} n_{3}} \exp \left\{-\left(u_{3}-u_{2}\right)\right\}- \\
& \frac{\left(n_{1}-1\right)(n 2-1)\left(n_{3}+2\right)}{3 n_{1} n_{2} n_{3}} \exp \left\{-\left(2 u_{3}-u_{2}-u_{1}\right)\right\} \\
I_{2}= & 1-\frac{\left(n_{3}-1\right)\left(n_{2}+1\right)}{2 n_{2} n_{3}} \exp \left\{-\left(u_{2}-u_{3}\right)\right\}+\frac{(n 3-1)\left(n_{1}-1\right)\left(n_{2}+2\right)}{6 n_{1} n_{2} n_{3}} \times \\
& \exp \left\{-\left(2 u_{2}-u_{1}-u_{3}\right)\right\}, \\
I_{3}= & 1-\frac{\left(n_{3}-1\right)\left(n_{1}+1\right)}{2 n_{1} n_{3}} \exp \left\{-\left(u_{1}-u_{3}\right)\right\}+\frac{(n 3-1)\left(n_{2}-1\right)\left(n_{1}+2\right)}{6 n_{1} n_{2} n_{3}} \times \\
& \exp \left\{-\left(2 u_{1}-u_{2}-u_{3}\right)\right\},
\end{aligned}
$$

and $U_{m}=\max \left(U_{1}, U_{2}, U_{3}\right)$. The following theorem will then provide the UMVUE of $R_{3}$.

Theorem 2. Based on the random samples $Y_{i 1}, Y_{i 2}, \cdots, Y_{i n_{i}}, i=1,2,3$ and setting $U_{i}=u_{i}, i=1,2,3$, $U_{m}=u_{m}$, the UMVUE of $R_{3}$ will be given by

$$
\widehat{R}_{3 U}=1-\widehat{\bar{R}}_{3 U}
$$

where

$$
\widehat{\bar{R}}_{3 u}=\left\{\begin{array}{l}
I_{1} \text { for } u_{m}=u_{3} \\
I_{2} \text { for } u_{m}=u_{2} \\
I_{3} \text { for } u_{m}=u_{1}
\end{array}\right.
$$

Proof. Let us define

$$
I=\left\{\begin{array}{l}
1 \text { if } Y_{31}>\max \left(Y_{11}, Y_{21}\right) \\
0 \text { otherwise }
\end{array}\right.
$$

Then, due to the fact that $\left(U_{1}, U_{2}, U_{3}\right)$ is a complete sufficient statistic for $\left(\mu_{1}, \mu_{2}, \mu_{3}\right)$ and $I$ is an unbiased estimator of $R_{3}, E\left[I \mid\left(u_{1}, u_{2}, u_{3}\right)\right]$ will be the UMVUE of $R_{3}$.

Therefore,

$$
\begin{aligned}
\widehat{R}_{3 U} & =E\left[I \mid\left(u_{1}, u_{2}, u_{3}\right)\right]=\operatorname{Pr}\left(Y_{31}>T_{1} \mid\left(u_{1}, u_{2}, u_{3}\right)\right) \\
& =1-\operatorname{Pr}\left(Y_{31} \leq T_{1} \mid\left(u_{1}, u_{2}, u_{3}\right)\right) \\
& =1-\widehat{\bar{R}}_{3 U} .
\end{aligned}
$$

Thus it suffices to find an explicit expression for $\widehat{\bar{R}}_{3 U}$. We employ Theorem 1 to get an expression of $\widehat{\bar{R}}_{3 U}$. Now, for the case $u_{m}=u_{3}$, we have

$$
\begin{aligned}
\widehat{\bar{R}}_{3 U} & =\operatorname{Pr}\left(Y_{31} \leq T_{1} \mid\left(u_{1}, u_{2}, u_{3}\right)\right)=\int \operatorname{Pr}\left(Y_{31} \leq t_{1} \mid u_{3}, T_{1}=t_{1}\right) f\left(t_{1} \mid\left(u_{1}, u_{2}\right)\right) d t_{1} \\
& =\int_{u_{3}}^{\infty} \operatorname{Pr}\left(Y_{31} \leq t_{1} \mid u_{3}\right) f\left(t_{1} \mid\left(u_{1}, u_{2}\right)\right) d t_{1} \\
& =\frac{\left(n_{1}-1\right)\left(n_{3}+1\right)}{2 n_{1} n_{3}} \exp \left\{-\left(u_{3}-u_{1}\right)\right\}+\frac{\left(n_{2}-1\right)\left(n_{3}+1\right)}{2 n_{2} n_{3}} \exp \left\{-\left(u_{3}-u_{2}\right)\right\}- \\
& \frac{\left(n_{1}-1\right)(n 2-1)\left(n_{3}+2\right)}{3 n_{1} n_{2} n_{3}} \exp \left\{-\left(2 u_{3}-u_{2}-u_{1}\right)\right\}=I_{1}
\end{aligned}
$$


For the case $u_{m}=u_{2}$, we have

$$
\begin{aligned}
\widehat{\bar{R}}_{3 U}= & \operatorname{Pr}\left(Y_{31} \leq T_{1} \mid\left(u_{1}, u_{2}, u_{3}\right)\right)=\int \operatorname{Pr}\left(Y_{31} \leq t_{1} \mid u_{3}, T_{1}=t_{1}\right) f\left(t_{1} \mid\left(u_{1}, u_{2}\right)\right) d t_{1} \\
= & \operatorname{Pr}\left(Y_{31} \leq t_{1} \mid u_{3}\right) \operatorname{Pr}\left(T_{1}=t_{1} \mid u_{1}, t_{1}=u_{2}\right)+ \\
& \int_{u_{2}}^{\infty} \operatorname{Pr}\left(Y_{3(1)} \leq t_{1} \mid u_{3}\right) f\left(t_{1} \mid\left(u_{1}, u_{2}\right)\right) d t_{1} \\
= & 1-\frac{\left(n_{3}-1\right)\left(n_{2}+1\right)}{2 n_{2} n_{3}} \exp \left\{-\left(u_{2}-u_{3}\right)\right\}+ \\
& \frac{(n 3-1)\left(n_{1}-1\right)\left(n_{2}+2\right)}{6 n_{1} n_{2} n_{3}} \exp \left\{-\left(2 u_{2}-u_{1}-u_{3}\right)\right\}=I_{2} .
\end{aligned}
$$

Finally, for the case $u_{m}=u_{1}$, we have

$$
\begin{aligned}
\widehat{\bar{R}}_{3 U}= & \operatorname{Pr}\left(Y_{31} \leq T_{1} \mid\left(u_{1}, u_{2}, u_{3}\right)\right)=\int \operatorname{Pr}\left(Y_{31} \leq t_{1} \mid u_{3}, T_{1}=t_{1}\right) f\left(t_{1} \mid\left(u_{1}, u_{2}\right)\right) d t_{1} \\
= & \operatorname{Pr}\left(Y_{31} \leq t_{1} \mid u_{3}\right) \operatorname{Pr}\left(T_{1}=t_{1} \mid t_{1}=u_{1}, u_{2}\right)+ \\
& \int_{u_{1}}^{\infty} \operatorname{Pr}\left(Y_{31} \leq t_{1} \mid u_{3}\right) f\left(t_{1} \mid\left(u_{1}, u_{2}\right)\right) d t_{1} \\
= & 1-\frac{\left(n_{3}-1\right)\left(n_{1}+1\right)}{2 n_{1} n_{3}} \exp \left\{-\left(u_{1}-u_{3}\right)\right\}+ \\
& \frac{(n 3-1)\left(n_{2}-1\right)\left(n_{1}+2\right)}{6 n_{1} n_{2} n_{3}} \exp \left\{-\left(2 u_{1}-u_{2}-u_{3}\right)\right\}=I_{3} .
\end{aligned}
$$

Hence proved.

\subsection{Bayes Estimator}

In this section we calculate Bayes estimator and generalized Bayes estimator of $R_{3}$ by plug-in method. For Bayes estimator, we consider the following independent priors

$$
f\left(\mu_{i}\right)=\exp \left\{-\left(\mu_{i}-\xi_{i}\right)\right\} ; \mu_{i}>\xi_{i},-\infty<\xi_{i}<\infty, i=1,2,3 .
$$

Theorem 3. Based on the independent priors stated in (5), the Bayes estimator of $R_{3}$ will be given by

$$
\widehat{R}_{3 B E}=\left(1-\widehat{R}_{1 B E}\right) I\left(\widehat{\mu}_{B E} \leq \widehat{\mu}_{3 B E}\right)+\left(1-\widehat{R}_{2 B E}\right) I\left(\widehat{\mu}_{B E}>\widehat{\mu}_{3 B E}\right),
$$

where $\widehat{\mu}_{i B E}, i=1,2,3$ and $\widehat{\mu}_{B E}$ are the Bayes estimators of $\mu_{i}, i=1,2,3$ and $\mu$ respectively.

Proof. Based on the independent priors stated in (5), we have

$$
f\left(u_{i} \mid \mu_{i}\right)=n_{i} \exp \left\{-n_{i}\left(u_{i}-\mu_{i}\right)\right\} ; u_{i}>\mu_{i},-\infty<\mu_{i}<\infty, i=1,2,3 .
$$

After some algebraic simplification, we get the posterior distribution of $\mu_{i}$ given $u_{i}$

$$
f\left(\mu_{i} \mid u_{i}\right)=\frac{\left(n_{i}-1\right) \exp \left\{\left(n_{i}-1\right) \mu_{i}\right\}}{\left[\exp \left\{\left(n_{i}-1\right) u_{i}\right\}-\exp \left\{\left(n_{i}-1\right) \xi_{i}\right\}\right]} ; \xi_{i}<\mu_{i}<u_{i}, i=1,2,3 .
$$

Under squared error loss, the Bayes estimator of $\mu_{i}(i=1,2,3)$ will be

$$
\widehat{\mu}_{i B E}=\frac{u_{i} \exp \left\{\left(n_{i}-1\right) u_{i}\right\}-\xi_{i} \exp \left\{\left(n_{i}-1\right) \xi_{i}\right\}}{\left[\exp \left\{\left(n_{i}-1\right) u_{i}\right\}-\exp \left\{\left(n_{i}-1\right) \xi_{i}\right\}\right]}-\frac{1}{\left(n_{i}-1\right)}
$$


Plugging $\widehat{\mu}_{i B E}(i=1,2,3)$, as describe in (6), in the expressions of $R_{1}, R_{2}$ and $\mu$ respectively, we get the following estimators

$$
\begin{aligned}
\widehat{R}_{1 B E}= & \frac{1}{2} \sum_{i=1}^{2} \exp \left\{-\left(\widehat{\mu}_{3 B E}-\widehat{\mu}_{i B E}\right)\right\}-\frac{1}{3} \exp \left\{-\left(2 \widehat{\mu}_{3 B E}-\hat{\mu}_{2 B E}-\hat{\mu}_{1 B E}\right)\right\} \\
\widehat{R}_{2 B E}= & \sum_{i=1}^{2} \exp \left\{-\left(\widehat{\mu}_{B E}-\widehat{\mu}_{i B E}\right)\right\}-\exp \left\{-\left(2 \widehat{\mu}_{B E}-\hat{\mu}_{2 B E}-\widehat{\mu}_{1 B E}\right)\right\} \\
& -\frac{1}{2} \exp \left\{-\left(2 \widehat{\mu}_{B E}-\widehat{\mu}_{1 B E}-\widehat{\mu}_{3 B E}\right)\right\}-\frac{1}{2} \exp \left\{-\left(2 \widehat{\mu}_{B E}-\widehat{\mu}_{2 B E}-\widehat{\mu}_{3 B E}\right)\right\} \\
& +\frac{2}{3} \exp \left\{-\left(3 \widehat{\mu}_{B E}-\widehat{\mu}_{1 B E}-\widehat{\mu}_{2 B E}-\widehat{\mu}_{3 B E}\right)\right\}
\end{aligned}
$$

and $\widehat{\mu}_{B E}=\max \left(\widehat{\mu}_{1 B E}, \widehat{\mu}_{2 B E}\right)$.

This completes the proof of Theorem 3.

For deriving the generalized Bayes estimator of $R_{3}$, we consider the following improper prior for each $\mu_{i}, i=1,2,3$.

$$
f\left(\mu_{i}\right)=1 ;-\infty<\mu_{i}<\infty, i=1,2,3
$$

Now, we have the following theorem which provides generalized Bayes estimator of $R_{3}$.

Theorem 4. Based on the independent priors stated in (7), the generalized Bayes estimator of $R_{3}$ will be given by

$$
\widehat{R}_{3 G B E}=\left(1-\widehat{R}_{1 G B E}\right) I\left(\widehat{\mu}_{G B E} \leq \widehat{\mu}_{3 G B E}\right)+\left(1-\widehat{R}_{2 G B E}\right) I\left(\widehat{\mu}_{G B E}>\widehat{\mu}_{3 G B E}\right),
$$

where $\widehat{\mu}_{i G B E}, i=1,2,3$ and $\widehat{\mu}_{G B E}$ are the generalized Bayes estimators of $\mu_{i}, i=1,2,3$ and $\mu$ respectively.

Proof. Based on the prior given in (7), the proof of Theorem 4 will follow along the line of the proof of Theorem 3. Here it is to be noted that the the generalized Bayes estimator of $\mu_{i}(i=1,2,3)$ will be

$$
\widehat{\mu}_{i G B E}=u_{i}-\frac{1}{n_{i}}
$$

Based on the above findings, we have the following notable remark.

Remark 1. For $i=1,2,3$, if we take the limit $n_{i} \rightarrow \infty$, then generalized Bayes estimator of $R_{3}$ will be limit of the Bayes estimator.

\section{Simulation Study}

In this section we compare the estimators of the reliability function $\left(R_{3}\right)$ through the risk values with respect to the following loss functions

$$
\begin{aligned}
& \text { Log Square Error Loss : } L_{1}\left(\hat{R}_{3}, R_{3}\right)=\left(\frac{\log \hat{R}_{3}-\log R_{3}}{\log R_{3}}\right)^{2}, \\
& \text { Linex Loss : } L_{2}\left(\hat{R}_{3}, R_{3}\right)=b\left(e^{a\left(\hat{R}_{3}-R_{3}\right)}-a\left(\hat{R}_{3}-R_{3}\right)-1\right) ; a \in \mathcal{R}, b>0, \text { and } \\
& \text { Square Error Loss : } L_{3}\left(\hat{R}_{3}, R_{3}\right)=\left(\hat{R}_{3}-R_{3}\right)^{2} .
\end{aligned}
$$

It is to be noted that it is difficult to get any closed form analytic solutions of the risk values for comparison of the different estimators. So that, for comparing the estimators we have used Monte Carlo simulation method with $N=5000$ replications. To evaluate Bayes estimator (BE) we have taken $\xi_{1}=\xi_{2}=\xi_{3}=1$. In the linex loss function we set $a=1, b=2$. On the basis of simulation study, we can 
draw the following conclusion. Table 1-2 show the risk value of the estimators under log square error loss function. The following points are clear from these Tables. If we take 0.5 as the base value for $\mu_{1}$ and increase it successively by 0.5 until it reaches 2 , the risk values decrease for different choices of $\mu_{2}$ and $\mu_{3}(0.5,1,1.5,2)$. It is observed that when $n_{1}=n_{2}=n_{3}=5$ the maximum likelihood estimator, Bayes estimator and generalized Bayes estimator are equivalent $(M L E \equiv B E \equiv G B E)$ and these estimators are better than the uniformly minimum variance unbiased estimator. For unequal sample sizes we can arrange the performance of the estimators as $B E \equiv G B E \geq U M V U E \geq M L E$. It is obvious that the risk values will decrease with the increase in the sample size. It is observed that the performance of the estimators are quite similar when comparison is made on the basis of the risk values under linex loss function and it is clear from Table 3-4.

Table 1. The risk values of different estimators under log square error loss function for the choices $n_{1}=5$, $n_{2}=5, n_{3}=5, \mu_{1}, \mu_{2}, \mu_{3}=0.5,, 1,1.5,2$.

\begin{tabular}{|c|c|c|c|c|c|c|}
\hline$\mu_{1}$ & $\mu_{2}$ & $\mu_{3}$ & MLE & GBE & BE & UMVUE \\
\hline 0.5000 & 0.5000 & 0.5000 & 0.0504 & 0.0504 & 0.0504 & 0.0569 \\
& 1.0000 & 1.0000 & 0.0646 & 0.0646 & 0.0646 & 0.0749 \\
& 1.5000 & 1.5000 & 0.0762 & 0.0762 & 0.0762 & 0.0913 \\
& 2.0000 & 2.0000 & 0.0956 & 0.0956 & 0.0956 & 0.1152 \\
\hline 1.0000 & 0.5000 & 0.5000 & 0.0340 & 0.0340 & 0.0340 & 0.0363 \\
& 1.0000 & 1.0000 & 0.0507 & 0.0507 & 0.0507 & 0.0573 \\
& 1.5000 & 1.5000 & 0.0621 & 0.0621 & 0.0621 & 0.0724 \\
& 2.0000 & 2.0000 & 0.0822 & 0.0822 & 0.0822 & 0.0977 \\
\hline 1.5000 & 0.5000 & 0.5000 & 0.0220 & 0.0220 & 0.0220 & 0.0228 \\
& 1.0000 & 1.0000 & 0.0340 & 0.0340 & 0.0340 & 0.0363 \\
& 1.5000 & 1.5000 & 0.0504 & 0.0504 & 0.0504 & 0.0569 \\
& 2.0000 & 2.0000 & 0.0646 & 0.0646 & 0.0646 & 0.0749 \\
\hline 2.0000 & 0.5000 & 0.5000 & 0.0148 & 0.0148 & 0.0148 & 0.0152 \\
& 1.0000 & 1.0000 & 0.0220 & 0.0220 & 0.0220 & 0.0228 \\
& 1.5000 & 1.5000 & 0.0340 & 0.0340 & 0.0340 & 0.0363 \\
& 2.0000 & 2.0000 & 0.0507 & 0.0507 & 0.0507 & 0.0573 \\
\hline
\end{tabular}

Table 2. The risk values of different estimators under log square error loss function for the choices $n_{1}=5$, $n_{2}=10, n_{3}=10, \mu_{1}, \mu_{2}, \mu_{3}=0.5,1,1.5,2$.

\begin{tabular}{|c|c|c|c|c|c|c|}
\hline$\mu_{1}$ & $\mu_{2}$ & $\mu_{3}$ & MLE & GBE & BE & UMVUE \\
\hline 0.5000 & 0.5000 & 0.5000 & 0.0276 & 0.0225 & 0.0225 & 0.0239 \\
& 1.0000 & 1.0000 & 0.0241 & 0.0214 & 0.0214 & 0.0226 \\
& 1.5000 & 1.5000 & 0.0247 & 0.0236 & 0.0236 & 0.0254 \\
& 2.0000 & 2.0000 & 0.0268 & 0.0262 & 0.0262 & 0.0286 \\
\hline 1.0000 & 0.5000 & 0.5000 & 0.0215 & 0.0178 & 0.0178 & 0.0183 \\
& 1.0000 & 1.0000 & 0.0260 & 0.0211 & 0.0211 & 0.0225 \\
& 1.5000 & 1.5000 & 0.0255 & 0.0224 & 0.0224 & 0.0237 \\
& 2.0000 & 2.0000 & 0.0250 & 0.0237 & 0.0237 & 0.0255 \\
\hline 1.5000 & 0.5000 & 0.5000 & 0.0148 & 0.0124 & 0.0124 & 0.0126 \\
& 1.0000 & 1.0000 & 0.0215 & 0.0178 & 0.0178 & 0.0183 \\
& 1.5000 & 1.5000 & 0.0260 & 0.0211 & 0.0211 & 0.0225 \\
& 2.0000 & 2.0000 & 0.0255 & 0.0224 & 0.0224 & 0.0237 \\
\hline 2.0000 & 0.5000 & 0.5000 & 0.0103 & 0.0086 & 0.0086 & 0.0087 \\
& 1.0000 & 1.0000 & 0.0148 & 0.0124 & 0.0124 & 0.0126 \\
& 1.5000 & 1.5000 & 0.0215 & 0.0178 & 0.0178 & 0.0183 \\
& 2.0000 & 2.0000 & 0.0260 & 0.0211 & 0.0211 & 0.0225 \\
\hline
\end{tabular}


Table 3. The risk values of different estimators under linex loss function for the choices $n_{1}=5, n_{2}=5, n_{3}=5$, $\mu_{1}, \mu_{2}, \mu_{3}=0.5,1,1.5,2$

\begin{tabular}{|c|c|c|c|c|c|c|}
\hline$\mu_{1}$ & $\mu_{2}$ & $\mu_{3}$ & MLE & GBE & BE & UMVUE \\
\hline 0.5000 & 0.5000 & 0.5000 & 0.0071 & 0.0071 & 0.0071 & 0.0081 \\
& 1.0000 & 1.0000 & 0.0083 & 0.0083 & 0.0083 & 0.0097 \\
& 1.5000 & 1.5000 & 0.0089 & 0.0089 & 0.0089 & 0.0107 \\
& 2.0000 & 2.0000 & 0.0103 & 0.0103 & 0.0103 & 0.0124 \\
\hline 1.0000 & 0.5000 & 0.5000 & 0.0050 & 0.0050 & 0.0050 & 0.0052 \\
& 1.0000 & 1.0000 & 0.0071 & 0.0071 & 0.0071 & 0.0081 \\
& 1.5000 & 1.5000 & 0.0079 & 0.0079 & 0.0079 & 0.0092 \\
& 2.0000 & 2.0000 & 0.0094 & 0.0094 & 0.0094 & 0.0112 \\
\hline 1.5000 & 0.5000 & 0.5000 & 0.0026 & 0.0026 & 0.0026 & 0.0024 \\
& 1.0000 & 1.0000 & 0.0050 & 0.0050 & 0.0050 & 0.0052 \\
& 1.5000 & 1.5000 & 0.0071 & 0.0071 & 0.0071 & 0.0081 \\
& 2.0000 & 2.0000 & 0.0083 & 0.0083 & 0.0083 & 0.0097 \\
\hline 2.0000 & 0.5000 & 0.5000 & 0.0011 & 0.0011 & 0.0011 & 0.0010 \\
& 1.0000 & 1.0000 & 0.0026 & 0.0026 & 0.0026 & 0.0024 \\
& 1.5000 & 1.5000 & 0.0050 & 0.0050 & 0.0050 & 0.0052 \\
& 2.0000 & 2.0000 & 0.0071 & 0.0071 & 0.0071 & 0.0081 \\
\hline
\end{tabular}

Table 4. The risk values of different estimators under linex loss function for the choices $n_{1}=5, n_{2}=10, n_{3}=10$, $\mu_{1}, \mu_{1}, \mu_{3}=0.5,1,1.5,2$

\begin{tabular}{|c|c|c|c|c|c|c|}
\hline$\mu_{1}$ & $\mu_{2}$ & $\mu_{3}$ & MLE & GBE & BE & UMVUE \\
0.5000 & 0.5000 & 0.5000 & 0.0029 & 0.0028 & 0.0028 & 0.0030 \\
& 1.0000 & 1.0000 & 0.0029 & 0.0028 & 0.0028 & 0.0030 \\
& 1.5000 & 1.5000 & 0.0030 & 0.0030 & 0.0030 & 0.0033 \\
& 2.0000 & 2.0000 & 0.0032 & 0.0032 & 0.0032 & 0.0035 \\
\hline 1.0000 & 0.5000 & 0.5000 & 0.0019 & 0.0020 & 0.0020 & 0.0019 \\
& 1.0000 & 1.0000 & 0.0028 & 0.0027 & 0.0027 & 0.0029 \\
& 1.5000 & 1.5000 & 0.0030 & 0.0029 & 0.0029 & 0.0031 \\
& 2.0000 & 2.0000 & 0.0030 & 0.0030 & 0.0030 & 0.0033 \\
\hline 1.5000 & 0.5000 & 0.5000 & 0.0009 & 0.0010 & 0.0010 & 0.0009 \\
& 1.0000 & 1.0000 & 0.0019 & 0.0020 & 0.0020 & 0.0019 \\
& 1.5000 & 1.5000 & 0.0028 & 0.0027 & 0.0027 & 0.0029 \\
& 2.0000 & 2.0000 & 0.0030 & 0.0029 & 0.0029 & 0.0031 \\
\hline 2.0000 & 0.5000 & 0.5000 & 0.0004 & 0.0004 & 0.0004 & 0.0004 \\
& 1.0000 & 1.0000 & 0.0009 & 0.0010 & 0.0010 & 0.0009 \\
& 1.5000 & 1.5000 & 0.0019 & 0.0020 & 0.0020 & 0.0019 \\
& 2.0000 & 2.0000 & 0.0028 & 0.0027 & 0.0027 & 0.0029 \\
\hline
\end{tabular}

In the Table 5-6 we have compared the risk value of the estimators under square error loss function. It is noticed that when $n_{1}=n_{2}=n_{3}=5$ then $M L E \equiv G B E$ and their risk values are very close for different sample sizes. According to this loss function $B E$ is better than $U M V U E$ and $U M V U E$ is better than $M L E$ and $G B E$. It is also observed that the Bayes estimator gives the best performance among all estimators. 
Table 5. The risk values of different estimators under square error loss function for the choices $n_{1}=5$, $n_{2}=5, n_{3}=5, \mu_{1}, \mu_{2}, \mu_{3}=0.5,1,1.5,2$.

\begin{tabular}{|c|c|c|c|c|c|c|}
\hline$\mu_{1}$ & $\mu_{1}$ & $\mu_{3}$ & MLE & GBE & BE & UMVUE \\
\hline 0.5000 & 0.5000 & 0.5000 & 0.0069 & 0.0069 & 0.0015 & 0.0078 \\
& 1.0000 & 1.0000 & 0.0082 & 0.0082 & 0.0048 & 0.0095 \\
& 1.5000 & 1.5000 & 0.0089 & 0.0089 & 0.0073 & 0.0106 \\
& 2.0000 & 2.0000 & 0.0102 & 0.0102 & 0.0094 & 0.0123 \\
\hline 1.0000 & 0.5000 & 0.5000 & 0.0048 & 0.0048 & 0.0042 & 0.0050 \\
& 1.0000 & 1.0000 & 0.0069 & 0.0069 & 0.0038 & 0.0078 \\
& 1.5000 & 1.5000 & 0.0078 & 0.0078 & 0.0062 & 0.0091 \\
& 2.0000 & 2.0000 & 0.0093 & 0.0093 & 0.0087 & 0.0111 \\
\hline 1.5000 & 0.5000 & 0.5000 & 0.0024 & 0.0024 & 0.0054 & 0.0024 \\
& 1.0000 & 1.0000 & 0.0048 & 0.0048 & 0.0039 & 0.0050 \\
& 1.5000 & 1.5000 & 0.0069 & 0.0069 & 0.0058 & 0.0078 \\
& 2.0000 & 2.0000 & 0.0082 & 0.0082 & 0.0077 & 0.0095 \\
\hline 2.0000 & 0.5000 & 0.5000 & 0.0010 & 0.0010 & 0.0033 & 0.0010 \\
& 1.0000 & 1.0000 & 0.0024 & 0.0024 & 0.0027 & 0.0024 \\
& 1.5000 & 1.5000 & 0.0048 & 0.0048 & 0.0044 & 0.0050 \\
& 2.0000 & 2.0000 & 0.0069 & 0.0069 & 0.0066 & 0.0078 \\
\hline
\end{tabular}

Table 6. The risk values of different estimators under square error loss function for the choices $n_{1}=5$, $n_{2}=5, n_{3}=5, \mu_{1}, \mu_{2}, \mu_{3}=0.5,1,1.5,2$.

\begin{tabular}{|c|c|c|c|c|c|c|}
\hline$\mu_{1}$ & $\mu_{2}$ & $\mu_{3}$ & MLE & GBE & BE & UMVUE \\
\hline 0.5000 & 0.5000 & 0.5000 & 0.0029 & 0.0028 & 0.0003 & 0.0030 \\
& 1.0000 & 1.0000 & 0.0029 & 0.0028 & 0.0027 & 0.0030 \\
& 1.5000 & 1.5000 & 0.0030 & 0.0030 & 0.0032 & 0.0033 \\
& 2.0000 & 2.0000 & 0.0032 & 0.0032 & 0.0033 & 0.0035 \\
\hline 1.0000 & 0.5000 & 0.5000 & 0.0019 & 0.0019 & 0.0035 & 0.0019 \\
& 1.0000 & 1.0000 & 0.0028 & 0.0026 & 0.0015 & 0.0028 \\
& 1.5000 & 1.5000 & 0.0030 & 0.0029 & 0.0026 & 0.0031 \\
& 2.0000 & 2.0000 & 0.0030 & 0.0030 & 0.0029 & 0.0032 \\
\hline 1.5000 & 0.5000 & 0.5000 & 0.0009 & 0.0010 & 0.0051 & 0.0009 \\
& 1.0000 & 1.0000 & 0.0019 & 0.0019 & 0.0017 & 0.0019 \\
& 1.5000 & 1.5000 & 0.0028 & 0.0026 & 0.0024 & 0.0028 \\
& 2.0000 & 2.0000 & 0.0030 & 0.0029 & 0.0028 & 0.0031 \\
\hline 2.0000 & 0.5000 & 0.5000 & 0.0004 & 0.0004 & 0.0033 & 0.0004 \\
& 1.0000 & 1.0000 & 0.0009 & 0.0010 & 0.0012 & 0.0009 \\
& 1.5000 & 1.5000 & 0.0019 & 0.0019 & 0.0020 & 0.0019 \\
& 2.0000 & 2.0000 & 0.0028 & 0.0026 & 0.0026 & 0.0028 \\
\hline
\end{tabular}

\section{References}

1. Ali, M. M., Woo, J., and Pal, M., "Inference on reliability $\operatorname{Pr}(Y<X)$ in two-parameter exponential distribution," International Journal of Statistical Science, vol.3, pp. 119-125, 2004.

2. Baklizi, A., "Estimation of $\operatorname{Pr}(X<Y)$ using record values in the one and two parameter exponential distributions, "Communications in Statistics Theory and Methods, vol.37, pp. 692-698, 2008 a.

3. Baklizi, A., "Likelihood and Bayesian estimation of $\operatorname{Pr}(X<Y)$ using lower record values from the generalized exponential distribution," Computational Statistics $\&$ Data Analysis vol.52, pp. 3468-3473, 2008b.

4. Eryilmaz, S., "Consecutive k-out-of-n: G system in stress-strength set up," Communication in Statistics-Simulation and Computation, vol.37, pp. 579-589, 2008a. 
5. Eryilmaz, S., "Multivariate stress strength reliability model and its evaluation for coherent structures," Journal of Multivariate Analysis, vol.99, pp. 1878-1887, 2008b.

6. Eryilmaz, S., "On system reliability in stress strength set up," Statistics and Probability Letters, vol.80, pp. 834-839, 2010.

7. Eryilmaz, S.,"A new perspective to stress-strength models," Annals of the Institute of Statistical Mathematics, vol.63, no.1, pp. 101-115, 2011.

8. Gupta, R.D. and Gupta, R.C., "Estimation of $\operatorname{Pr}\left(Y_{p}>\max \left(Y_{1}, Y_{2}, \cdots, Y_{p-1}\right)\right)$ in the exponential case," Communication in Statistics-Theory and Methods, vol.17, pp. 911-924, 1988.

9. Ivshin, V. V., "Unbiased estimation of $P(X<Y)$ and their variances in the case of uniform and two-parameter distributions," J.Math.Sci, vol.81, pp. 2790-2793, 1996.

10. Kotz, S., Lumelsdii,Y. and Pensky, M., "The Stress Strength Model and its Generalization," World Scientific, Singapore, 2003.

11. Kundu, D., Raqab , M.Z., "Estimation of $R=\operatorname{Pr}(Y<X)$ for three-parameter Weibull distribution," Statistics \& Probability Letters, vol.79, pp. 1839-1846, 2009.

12. Kundu, P., Kumar, S. and Chatterjee, K., "Estimating the Reliability Function," Calcutta Statistical Association Bulletin, vol.67, pp. 267-268, 2015.

13. Krishnamoorthy, K., Mukherjee, S. and Guo, H., "Inference on reliability in two-parameter exponential stress-strength model," Metrika, vol.65, pp. 261-273, 2007.

14. Krishnamoorthy, K., Lin, Y. and Xia, Y., "Confidence limits and prediction limits for a Weibull distribution based on the generalized variable approach," Journal of Statistical Planning and Inference, vol.139, pp.2675-2684, 2009.

15. Krishnamoorthy, K. and Lin, Y., "Confidence limits for stress-strength reliability involving Weibull models," Journal of Statistical Planning and Inference, vol.140, pp. 1754-1764, 2010.

16. Pal, M., Ali, M. M., and Woo, J., "Estimation and testing of $P(Y>X)$ in two-parameter exponential distributions," Statistics, vol.39, pp. 415-428, 2005.

17. Rao, G. S., "Estimation of reliability in multicomponent stress-strength model based on generalized exponential distribution," Colombian Journal of Statistics, vol.35, pp. 67-76, 2012.

18. Rao, G. S., "Estimation of reliability in multicomponent stress-strength based on inverse exponential distribution," International Journal of Statistics and Economics, vol.10, pp. 28-37, 2013.

19. Rezaei, S., Tahmasbi, R. and Mahmoodi, M., "Estimation of $\operatorname{Pr}(Y<X)$ for generalized Pareto distribution," Journal of Statistical Planning and Inference, vol.140, pp. 480-494, 2010.

20. Rinco, S., "Estimation of $\operatorname{Pr}\left(Y_{p}>\max \left(Y_{1}, Y_{2}, \cdots, Y_{p-1}\right)\right)$ : Predictive approach in exponential case," The Canadian Journal of Statistics, vol.11, no.3, pp. 239-244, 1983.

21. Sengupta, S., "Unbiased variance estimation in estimating $P(X>Y)$ for one and two parameter exponential populations," Metron-International Journal of Statistics, vol.LXVI, pp. 329-339, 2008. 